\title{
Sensitivity of adjustment to response-parameter correlations
}

\author{
J.J. Wagschal and Y. Yeivin \\ Racah Institute of Physics, Hebrew University of Jerusalem, 91904 Jerusalem, Israel
}

\begin{abstract}
The formalism of Least-Squares Adjustment enables treatment of response-parameter correlations. We study the effects of such correlations on the adjusted parameters and responses and on their associated uncertainty matrices. And in order to bracket these effects and avoid unnecessary technical complications, we limit our discourse to an elementary one-parameter one-response criticality problem.
\end{abstract}

\section{Introduction}

The formalism of the generalized Least-Squares Cross Section Adjustment methodology enables exploration of responseparameter correlations. The pertinent explicit prescriptions for the adjusted parameters and the adjusted responses and for the uncertainty matrices respectively associated with these two data sets, as well as for their cross-covariance matrix, were already derived in 1980 [1], and later applied to demonstrate that there was no point in trying to iterate an adjustment. In other words, re-adjusting the adjusted parameters by the adjusted responses, with the two data sets evidently correlated, one realizes that, in as much as the sensitivity matrix evaluated at the point in the parameter space representing the original parameter library and that matrix evaluated at the point representing the adjusted library are close enough, the re-adjusted parameters are practically identical to the adjusted ones [2]. The complete adjustment formulary (including response-parameter correlations) was further applied in an investigation of adjustment iterations in cases for which the linearity assumption fails [3].

As far as routine (neutron cross section related) applications are concerned, there has indeed been no need to use the complete formulary, since the generally available evaluated cross section libraries were entirely based on differential cross section measurements (and on nuclear reaction models). In fact, the evaluators, as a matter of principle, have always been very careful to avoid "contamination" of their libraries by any integral-measurement information [4]. These libraries have thus been manifestly uncorrelated to the integral responses by which they were supposed to be adjusted. Recall that, even for the simplest critical assemblies, the deviations of the calculated reactivities from their respective measured values, in general, were considerably greater than the uncertainties in the latter. This seems to have been the case until the very recent pre-release versions, and the formal initial release of ENDF/B-VII.0 [5]. Results of an MCNP criticality validation for 31 benchmarks using this nuclear data library [6] show an incredible agreement between the calculated and the measured reactivities: 19 of the 31 results fall within a single standard deviation of the corresponding benchmark value for $\mathrm{k}_{\mathrm{eff}}$, and only 6 differ by more than two standard deviations.
Incidentally, such an amazing agreement is only part of the story, for as important as reproducing a nominal value might be, the quality of a parameter library is also judged by the magnitude of the uncertainty in the calculated value that it predicts. Anyway, this "improvement", though not a result of a rigorous adjustment exercise, clearly indicates that what is known as "tuning" has been applied to the cross sections in the evaluation process. Such tuning manifestly introduces response-parameter correlations. However, since it is an art rather than a science, it is difficult, if not impossible, to quantify the response-parameter correlations it introduces.

We propose to examine the formalism and estimate the effect of such correlations on the adjusted parameters and responses, and on the uncertainties in these quantities. And in order to lay stress on this effect, and avoid unnecessary technical complications, we will limit our discussion to a simple one-parameter/one-response criticality problem.

\section{Notation and general prescriptions}

But first, for the sake of completeness and in order to introduce what we believe is a convenient notation, let us spell out the adjustment formulary. We consider a given library of $N$ parameters that we denote by $p \equiv\left\{p_{n}\right\}$ and a set of $I$ responses of which the measured values are $r \equiv\left\{r_{i}\right\}$ and the calculated values of which are functions of the parameters, that we denote by $\bar{r}=\bar{r}(p)$. The $N \times N$ uncertainty (variance-covariance) matrix associated with the parameter library is $C_{p}=\left\langle\delta p \delta p^{\dagger}\right\rangle$, where the dagger superscript signifies transposition, and the $I \times I$ uncertainty matrix associated with the measured response set is $C_{r}$. The $I \times N$ sensitivity matrix of the responses with respect to the parameters is $S_{i n}=\partial \bar{r}_{i} / \partial p_{n}$, which evidently means that $\delta \bar{r}=S \delta p$. Let us also consider the deviations of the calculated responses from their respective measured values, i.e., the vector $d=\bar{r}(p)-r$. This vector, in a certain sense, is the essence of adjustment, for apparently if $d \approx 0$ there is no need to adjust the parameters. The uncertainty matrix associated with the deviation vector is clearly

$$
\begin{aligned}
C_{d} & =\left\langle\delta(\bar{r}-r) \delta(\bar{r}-r)^{\dagger}\right\rangle \\
& =S C_{p} S^{\dagger}-S C_{p r}-C_{r p} S^{\dagger}+C_{r}
\end{aligned}
$$


where the nature of the $N \times I$ matrix $C_{p r}$ and that of its transpose $C_{r p}$ should be obvious. As we will soon realize, the matrix $C_{d}$ is also of central importance in the formulary. In particular, the uncertainty matrices associated with the adjusted quantities (which are independent of the value of the vector $d$ ) pivot on the inverse of this matrix.

With these preliminaries we can now write down the explicit prescriptions for the adjustment of a given library by a given set of measured responses. The adjusted parameters and the adjusted responses are given by

$$
\left.\begin{array}{l}
p^{\prime}=p+\left(C_{p r}-C_{p} S^{\dagger}\right) C_{d}^{-1} d \\
r^{\prime}=r+\left(C_{r}-C_{r p} S^{\dagger}\right) C_{d}^{-1} d
\end{array}\right\},
$$

the respective uncertainty matrices associated with the adjusted quantities are

$$
\left.\begin{array}{c}
C_{p^{\prime}}=C_{p}-\left(C_{p r}-C_{p} S^{\dagger}\right) C_{d}^{-1}\left(C_{r p}-S C_{p}\right) \\
C_{r^{\prime}}=C_{r}-\left(C_{r}-C_{r p} S^{\dagger}\right) C_{d}^{-1}\left(C_{r}-S C_{p r}\right)
\end{array}\right\},
$$

and the cross uncertainty (rectangular) matrix of the adjustedparameters and the adjusted responses is

$$
C_{p^{\prime} r^{\prime}}=C_{p r}-\left(C_{p r}-C_{p} S^{\dagger}\right) C_{d}^{-1}\left(C_{r}-S C_{p r}\right) .
$$

\section{The one-parameter/one-response case: the reduced prescriptions}

For the case of $N=I=1$ all vectors and matrices just degenerate to single numbers. Thus $C_{p}=\operatorname{var}(p)=\sigma_{p}^{2}$, and $C_{r}=\operatorname{var}(r)=\sigma_{r}^{2}$. Further, $C_{p r}=C_{r p}=\operatorname{cov}(p, r)=\rho \sigma_{p} \sigma_{r}$, where $\rho$ is the correlation coefficient and the $\sigma^{\prime} s$ are the respective standard deviations. Before discussing our actual problem, let us just mention that it will be a one-group oneregion "reactor" problem, and the one response as a function of the single parameter will be $\bar{r}(p)=p S$, with $\mathrm{S}$ the constant sensitivity $d \bar{r} / d p$.

With the above notation the deviation reduces to $d=p S-$ $r$, and its associated uncertainty matrix to

$$
\begin{aligned}
C_{d} & =S^{2} \sigma_{p}^{2}-2 \rho S \sigma_{p} \sigma_{r}+\sigma_{r}^{2} \\
& =\left(S \sigma_{p}-\rho \sigma_{r}\right)^{2}+\left(1-\rho^{2}\right) \sigma_{r}^{2}
\end{aligned}
$$

The adjusted parameter and the adjusted response, respectively, reduce to

$$
\left.\begin{array}{c}
p^{\prime}=p+\left(\rho \sigma_{r}-S \sigma_{p}\right) \frac{\sigma_{p} d}{C_{d}} \\
r^{\prime}=r+\left(\sigma_{r}-\rho S \sigma_{p}\right) \frac{\sigma_{r} d}{C_{d}}
\end{array}\right\} .
$$

The variance of the adjusted parameter, after some straightforward algebra, reduces to

$$
\sigma_{p^{\prime}}^{2}=\left(1-\rho^{2}\right) \frac{\sigma_{p}^{2} \sigma_{r}^{2}}{C_{d}} .
$$

The variance of the adjusted response could be similarly derived. However, this is unnecessary, since $\delta r^{\prime}=S \delta p^{\prime}$, from which it follows that

$$
\sigma_{r^{\prime}}^{2}=S^{2} \sigma_{p^{\prime}}^{2}
$$

By the same token, the covariance of the adjusted parameter and the adjusted response is

$$
\rho^{\prime} \sigma_{p^{\prime}} \sigma_{r^{\prime}}=S \sigma_{p^{\prime}}^{2}
$$

Note that considering the last two equations one realizes that, whatever the given value of the correlation coefficient $\rho$, the adjusted parameter and the adjusted response are always fully correlated, i.e., $\rho^{\prime}=1$. Note also that for $\rho= \pm 1$ the variances of both adjusted quantities vanish.

\section{A representative problem: the one-group bare multiplying sphere}

In the one-group isotropic scattering approximation of the neutron transport equation, two parameters determine the solutions of the equation for homogeneous uniform-density bare spheres. These parameters might well be the macroscopic total cross section $\Sigma$ and the average collision multiplication

$$
c=\frac{\bar{v} \sigma_{f}+2 \sigma_{n, 2 n}+3 \sigma_{n, 3 n}+\cdots+\sigma_{s}}{\sigma_{t o t}} .
$$

This parameter is as good a parameter as the more common average fission multiplication, $\eta=\bar{v} \sigma_{f} / \sigma_{a}$, as an indicator of the reactivity of a multiplying medium. And while the latter parameter is associated with the eigenvalue $k$ in the representation

$$
\Omega \cdot \nabla \psi+\Sigma \psi=S_{s}[\psi]+\frac{1}{k} S_{f}[\psi]
$$

of the stationary transport equation, the parameter $c$ is associated with $\gamma$ in the representation

$$
\Omega \cdot \nabla \psi+\Sigma \psi=\frac{1}{\gamma}\left\{S_{s}[\psi]+S_{f}[\psi]\right\} .
$$

Evidently, just as $k_{\infty}=\eta$, so $\gamma_{\infty}=c$.

There are several reasons to prefer $c$ to $\eta$. In the context of our discourse, however, the decisive argument in favour of $c$ is that, for a critical sphere, the collision multiplication, in fact, is the inverse of the non-leakage probability $P$ (i.e., the probability to make the next collision in the sphere), for clearly the condition for criticality is $c P=1$. In any event, it is plain to see that for any bare sphere, of which the medium is characterized by a given value of $\mathrm{c}$, the effective collision multiplication, or reactivity, is $\gamma=c P$. This conforms to the model that we have set out to explore in section 3 , namely $\bar{r}=p S$.

An interesting remark relating to our "physical" model is the following. The geometry of a bare homogeneous sphere is characterized by its radius in mean-free-paths. Whence a universal relation can be easily calculated of the non-leakage 


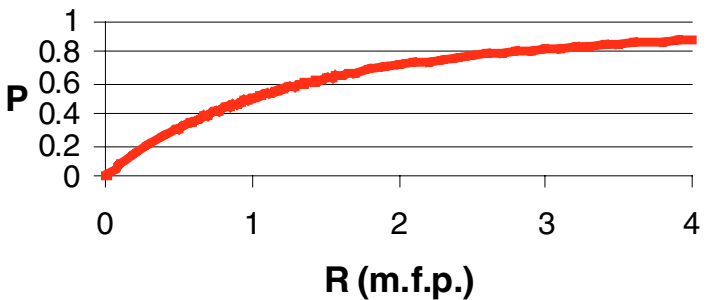

Fig. 1. The universal one-group isotropic scattering $P$ vs. $\Sigma R$ curve (ref. 7).

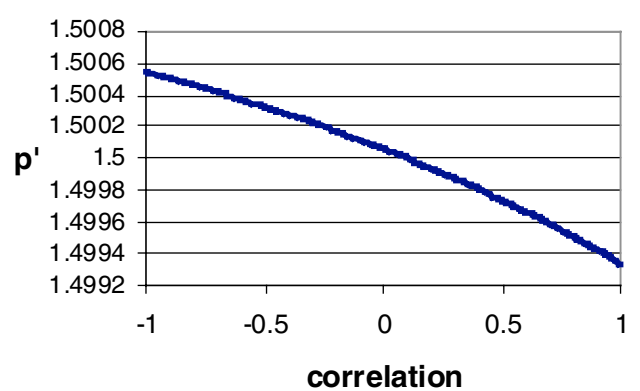

Fig. 2. The adjusted parameter as a function of the assumed correlation coefficient.

probability as a function of the radius (in m.f.p.) $\Sigma R$. This has indeed been done in ref. [7], and is reproduced here, as figure 1, in graphic form. We may also add that for realistic, multi-group non-isotropic scattering, bare spheres the graph is still valid, provided both $c$ and $\Sigma$ are the respective transport corrected, properly averaged one-group values.

Now, all things considered, we propose to study the effects of possible response-parameter correlations in the case of the critical bare sphere for which the non-escape probability is $P=2 / 3$ (and for which the corresponding radius, by the above universal relation, is $\Sigma R \sim 1.7$ ). Obviously then the average collision multiplication is $c=1.5$ and the reactivity $\gamma=1$. However, let us assume that the given parameter is $p=1.506 \pm 0.015$, resulting in a calculated reactivity $\bar{r}=\bar{\gamma}$ $=c P=1.506 \times \frac{2}{3}=1.004$, and it is to be adjusted by the "measured" response $r=1.000 \pm 0.001$. In order to evaluate the adjusted parameter and response, and the standard deviations of the adjusted parameter and of the adjusted response, we still need the sensitivity, which evidently is just $S=2 / 3$.

\section{Results and discussion}

The results of the straightforward calculations are all presented in graphic form, as each quantity is given as a function of the response-parameter correlation coefficient $\rho$. Thus figure 2 depicts $p^{\prime}$, the adjusted parameter, as a function of $\rho$.

Ideally one would like the adjusted parameter to be $p^{\prime}=$ 1.5 , the collision multiplication of the critical bare sphere for which $P=2 / 3$. We observe that indeed, for the whole range of $\rho$, the value of $p^{\prime}$ is within 0.0006 of its desired value. Compared to the given uncertainty 0.015 in $\mathrm{p}$, this is a remarkable result. Incidentally, the ideal adjustment result $p^{\prime}=1.5$ is obtained for $\rho=0.1$. The reason for this will become clear when we discuss the uncertainty in $r^{\prime}$. The plot

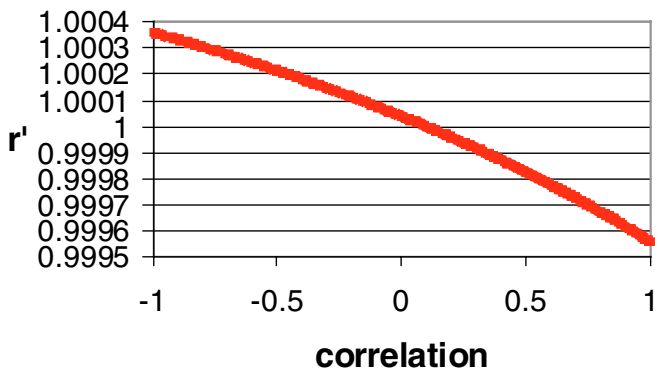

Fig. 3. The adjusted response as a function of the assumed correlation coefficient.

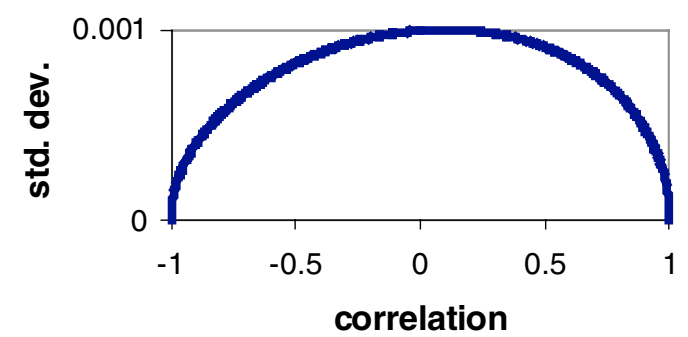

Fig. 4. The adjusted response standard deviation as a function of the assumed correlation coefficient.

of $r^{\prime}$ vs. $\rho$ is given in figure 3. Again, we realize that, for all values of $\rho$, the values of $r^{\prime}$ are within 0.0004 of the desired $r^{\prime}=1$, which is also obtained for $\rho=0.1$. And again this should be compared to the uncertainty 0.001 in $r$. The next figure, figure 4, shows the standard deviation of the adjusted response. We notice that the uncertainty in the adjusted response, as is to be expected, is indeed less than or equal to the uncertainty in the "given" measured response. And to find the value of $\rho$ for which the maximal uncertainty is obtained, we have to differentiate its explicit expression with respect to $\rho$, and find the value of $\rho$ for which the derivative vanishes. This is a straightforward matter, although somewhat convoluted. In any event, the derivative of $\sigma_{r^{\prime}}^{2}$ with respect to $\rho$ vanishes (in the range $-1 \leq \rho \leq 1$ ) at

$$
\rho=\frac{\sigma_{r}}{\sigma_{p} S}=\cdots=\frac{\tilde{\sigma}_{r}}{\tilde{\sigma}_{p}} \frac{r}{\bar{r}} .
$$

For the problem that we study: $r / \bar{r} \simeq 1$, and the relative standard deviations are $\tilde{\sigma}_{r}=0.1 \%$ and $\tilde{\sigma}_{p}=1 \%$, so that the desired value of the correlation coefficient is indeed $\rho \approx 0.1$. When we evaluate $p^{\prime}$ and $r^{\prime}$ for this value of $\rho$, we respectively obtain 1.5 and 1.0. Obviously, at this value of $\rho, \sigma_{r}-\rho S \sigma_{p}=0$, so that, by equation (6), $r^{\prime}=r$ and for a critical system is equal to 1 .

Finally, figure 5 displays five graphs of the standard deviations of the adjusted response for five different values of the (relative) uncertainty in the given response, in the range 0.1 to $1.0 \%$. We see that in each case $\tilde{\sigma}_{r^{\prime}} \leq \tilde{\sigma}_{r}$, and that $\tilde{\sigma}_{r^{\prime}, \max }=\tilde{\sigma}_{r}$ obtains for $\rho \approx \tilde{\sigma}_{r} / \tilde{\sigma}_{p}$.

\section{Summary and further comments}

We have studied adjustment in the elementary case of the onegroup bare critical sphere, and demonstrated that even when 


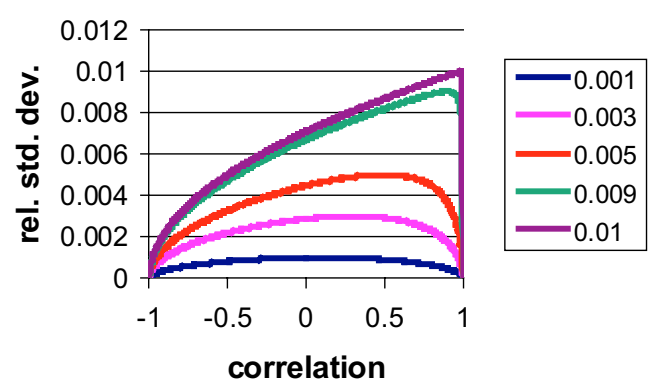

Fig. 5. The standard deviations of the adjusted response for five cases of different uncertainty in the given response. The parameter uncertainty is the same for all cases $\tilde{\sigma}_{p}=1 \%$. The lower graph is a reproduction of figure 4 , i.e., with $\tilde{\sigma}_{r}=0.1 \%$, and the other four graphs, in increasing order, respectively correspond to relative standard deviations of $0.3,0.5,0.9$, and $1 \%$.

response-parameter correlation is known to be present, but is quantitatively unknown, adjustment is still advisable and practical. Considering that the main purpose of adjustment is to generate parameters leading to more reliable calculations, i.e., reducing uncertainties in calculated responses, we have again shown that, to the extent that the measured responses are accurate (compared to the reliability of the calculated responses), and whatever the assumed correlations are, adjustment will result in better libraries.
We have also elaborated the fact that uncertainties in the adjusted responses are always smaller than or equal to the uncertainties in the given measured responses. It is important to note that even if additional responses, included in a given set, may not affect the nominal values of the adjusted parameters, they generally result in further reducing the uncertainties in the adjusted parameters and responses.

\section{References}

1. J. Barhen, J.J. Wagschal, Y. Yeivin, Trans. Am. Nucl. Soc. 35, 246 (1980).

2. R.L. Perel, J.J. Wagschal, Y. Yeivin, in Reactor Dosimetry: Radiation Metrology and Assessment, ASTM STP 1398, edited by J.G. Williams et al. (ASTM West Conshohoken, PA, 2001), p. 486.

3. J.J. Wagschal, Y. Yeivin, in Proc. Int. Conf. on Nuclear Data for Science and Technology ND 2001, edited by K. Shibata (J. Nucl. Sci. Tech., Supplement 2, 2002), p. 896.

4. S. Pearlstein (private communication) (1973).

5. M.B. Chadwick et al., Nucl. Data Sheets 107, 2931 (2006).

6. R.D. Mosteller, Los Alamos National Laboratory Report No. LAUR-07-0362, 2007.

7. R.L. Perel, J.J. Wagschal, Y. Yeivin, in Proc. Sixth Int. Conf. on Nuclear Criticality Safety, edited by P. Cousinou (Versailles, France, 1999), p. 1462. 